\title{
Contribution A L'analyse De L'interface Strategie- Demarche Qualite Des Entreprises Marocaines Certifiees ISO 9001
}

\author{
Mohamed Amine M'Barki \\ Ecole Nationale de Commerce et de Gestion Tanger- \\ Université Abd El Malek Essaadi \\ Professeur de l'enseignement supérieur département management, Morocco \\ Sara Rbili \\ Ecole Nationale de Commerce et Gestion - Tanger, Morocco \\ Doctorante en sciences de gestion
}

Doi:10.19044/esj.2018.v14n28p270 URL:http://dx.doi.org/10.19044/esj.2018.v14n28p270

\begin{abstract}
The purpose of this paper is to clarify the relation between the strategic reflection and the quality process through a detailed analysis of the contributions of these two fields in a specific context which is the ISO 9001 certified Moroccan companies.

Based on the organizational theories, the strategic schools, the key concepts of the TQM, and the contributions of 2015 version of the ISO 9001 standards we have proposed a performance-based unified theoretical integrative framework. From a methodological point of view, the coherence between the problematic and the data to which we have access and the nature of the studied phenomenon, has led us to prioritize a multi-method and sequential research approach.

We have chosen to begin our empirical study by an exploratory study that was conducted through the use of an interview guide delivered to a sample of experts. This resulted in a more rewarding research model which in turn was subjected to tests by using structural equations as a part of the second empirical phase which was conducted on a sample of ISO 9001 certified Moroccan companies.

The research has shed light on the emergence of a well differentiated typology of quality related behavior and a quite elaborated strategic reflection process. We have also confirmed the existence of an significant bilateral relation between the strategy and the quality, in accordance with an iterative process combining other factors (internal and external) that influence their transformation and their consequences. We have also highlighted the partial
\end{abstract}


nature of the mediator role in the improvement of the performance concerning the alignment of the two disciplines.

Keywords: Quality approach, business strategy, Performance, Competitive advantage, QMS.

\section{Résumé}

Cette recherche a pour objectif de clarifier la relation stratégie-qualité à travers une analyse approfondie des apports des deux disciplines dans un contexte plus spécifique qui est celui des entreprises marocaines certifiées ISO 9001.

En nous fondant sur les théories des organisations, les grands courants stratégiques, les principes fondateurs du TQM et sur les apports de la version 2015 de la norme ISO 9001, nous avons proposé un cadre théorique intégratif unifié basé sur la notion de performance. D'un point de vue méthodologique, la cohérence entre la problématique, les données auxquelles nous avons accès et la nature du phénomène étudié, nous ont conduits à privilégier une démarche de recherche multi-méthode et séquentielle. Nous avons choisi ainsi d'entamer notre investigation par une étude qualitative exploratoire, menée grâce à un guide d'entretien administré auprès d'experts afin d'enrichir notre modèle de recherche, qui va faire l'objet de tests au moyen d'équations structurelles lors de la seconde phase quantitative menée auprès d'un échantillon d'entreprises marocaines certifiées ISO 9001.

La recherche a mis en relief l'émergence d'une typologie bien différentiée des comportements qualité, et d'un processus de réflexion stratégique assez élaboré. Aussi, nous avons confirmé l'existence d'une relation réciproque très significative entre la stratégie et la qualité selon un processus itératif intégrant d'autres facteurs (internes et externes) qui influencent leur transformation et leurs conséquences. Nous avons aussi mis en évidence le caractère partiel du rôle médiateur de l'amélioration de la performance dans le rapprochement des deux disciplines.

Mots-clés : Démarche qualité, stratégie d'entreprise, Performance, Avantage concurrentiel, SMQ.

\section{Introduction:}

Le monde des affaires a subi ces dernières années des mutations profondes. Le rythme accéléré des changements a entraîné une forme d'incertitude obligeant les entreprises à développer une grande capacité d'innovation et d'adaptation à l'égard d'un environnement économique de plus en plus turbulent, marqué par l'accroissement de la compétitivité et par une diversification accrue des produits. 
Les entreprises sont alors appelées à maitriser et améliorer les performances de l'ensemble de leurs processus afin de garantir leur pérennité, en se fixant des stratégies plus poussées en termes de qualité, d'innovation et de flexibilité. Cela nécessite une bonne maitrise organisationnelle et technologique. Dans ce sens, toutes les entreprises reconnaissent à la qualité le vecteur incontournable de compétitivité et la variable stratégique de survie.

La qualité trouve désormais de plus en plus sa place, même au niveau de gestion le plus élevé dans l'organisation : celui de la stratégie d'entreprise. Bien sûr, la pensée stratégique a significativement évolué pour prendre en compte des besoins nouveaux. Cependant, la stratégie ne s'est pas encore suffisamment inspirée des apports potentiels, que peut lui procurer une meilleure intégration de la qualité.

Ainsi, les nouveaux apports de la version 2015 de la norme ISO 9001, qui intègrent explicitement l'aspect stratégique dans la conduite d'un projet qualité, soulèvent un questionnement très actuel : Quel impact réciproque entre la réflexion stratégique de l'entreprise et la démarche qualité ? Et sous quelles conditions?

La nature de l'interface stratégie-qualité est alors peu connue et relativement nouvelle. La complexité des liens à effectuer lors de l'enquête et les multiples variables contextuelles qui pourraient les affecter, à notre point de vue, nécessitent l'utilisation d'une démarche multi-méthodes. A cet effet, nous avons entamé cette recherche par une phase exploratoire à la fois théorique et empirique : l'exploration théorique a permis d'opérer un lien entre les courants stratégiques et les apports en matière des systèmes qualité. L'exploration empirique quant à elle a permis de mieux appréhender la réalité des deux disciplines ainsi que les modalités de leurs intersections en contexte marocain. Les résultats de cette double exploration ont débouché sur l'expression plus précise de la problématique en limitant notre champ d'investigation aux entreprises disposant d'un système de management qualité et en introduisant l'amélioration de la performance comme variable médiatrice dans cette relation. Le modèle de recherche élaboré à l'issue de cette première phase qualitative a été testé et validé grâce à une seconde étape empirique quantitative.

\section{Cadre théorique : Un renouveau des approches}

$\mathrm{Au}$ fil du temps, la stratégie et la qualité ont connu une évolution incessante et parfois radicale depuis leurs apparitions. Ce développement trace un parallélisme entre les deux disciplines puisqu'elles ont tenté de répondre à des problématiques identiques qui ont marqué l'époque en question. Toutefois, un rapprochement théorique entre la stratégie et la qualité en tant que corpus unifié n'est observé que tout récemment notamment dans le cadre de la version 2015 de la norme ISO 9001. 
Néanmoins, étudier l'interface des deux disciplines présente encore beaucoup d'ambiguïtés aussi bien au niveau théorique qu'au niveau managérial. D'une part, la qualité et la stratégie sont deux champs multidimensionnels distincts et l'étude de leur relation exige la prise en compte simultanée des relations entre les démarches et les activités qui les composent. D'autre part, organiser la démarche qualité de manière efficace et en harmonie avec la stratégie générale, exige de concilier des perspectives nombreuses et souvent contradictoires; la stratégie étant le résultat d'un processus de négociations inter-fonctionnelles, alors que la démarche qualité répond à des exigences figées par des normes.

\subsection{La démarche qualité et stratégie d'entreprise : une relation controversée}

La problématique de l'interface stratégie-qualité suscite encore l'engouement des chercheurs et des praticiens, du fait que malgré des contributions indéniables à la réflexion organisationnelle (Benezech D., \& Loos-Baroin J.(2003), Chemangui, M. (2009), Khetib, M. \& Ghomari, S. (2010), Svetoslav Georgiev \& Emil Georgiev (2015)), la démarche qualité perd progressivement sa consécration dans l'entreprise, elle n'arrive pas à trouver sa juste place au sein des organisations en raison de son incapacité à intégrer la perspective stratégique.

Ce constat d'échec des promesses de la qualité est dû entre autre à l'augmentation des coûts engendrés par la fonction qui ne se traduit pas forcément par une augmentation proportionnelle des bénéfices. Les praticiens de la qualité s'estiment plus concernés par les performances commerciales et n'ont pas le recul suffisant pour mesurer l'impact financier de leurs décisions. En effet, pour la recherche d'un avantage concurrentiel, l'orientation client ne peut plus à elle seule dominer la réflexion stratégique, une attention particulière doit être aussi portée aux autres parties prenantes, notamment : les actionnaires plus intéressés par le court terme et les performances financières. Dans ce sens, la qualité ne joue qu'un rôle périphérique dans l'analyse stratégique.

Aussi, l'émergence du management stratégique, ainsi que le fait qu'on prêtait à la qualité plutôt un rôle de fonction de support et une vision de court terme ont été deux des nombreux facteurs contribuant au refoulement de la discipline au second plan avant cette période. Ce peu intérêt stratégique accordé à la fonction qualité est dû au fait que la qualité a fait beaucoup d'apports pratiques, mais peu de contributions théoriques. De plus, la qualité se concrétise essentiellement lors de la définition de la démarche à poursuivre, alors que les orientations de la politique qualité peuvent être précieuses au niveau même de la formulation stratégique.

En outre, on observe dans certains cas une incohérence entre les missions de l'entreprise, et les objectifs tracés dans sa politique qualité, chose 
qui empêche la démarche qualité de devenir une philosophie dominante pour l'entreprise.

Ces recoupements potentiels entre qualité et stratégie peuvent laisser des illusions relatives à une éventuelle compétition entre les deux disciplines pour améliorer la performance de l'entreprise. Et même s'il est vrai que la stratégie domine toute les autres fonctions de l'entreprise même celle de la qualité, il est néanmoins nécessaire de redonner à la démarche qualité au moins le titre de force motrice de la stratégie (Siegel, D. \& Roesslinge, F) .

\subsection{Démarche qualité et la stratégie d'entreprise: Un rapprochement historique et conceptuel}

Retracer les évolutions de la qualité et de la stratégie nous amène à nous positionner davantage dans une optique de rapprochement chronologique et à retracer les échanges entre les deux disciplines.

Tableau 1 : Le développement de la pensée stratégique et des démarches qualité au fil des mutations de l'environnement des entreprises

\begin{tabular}{|c|c|c|c|}
\hline Période & Caractéristiques de l'environnement & $\begin{array}{l}\text { Démarche } \\
\text { qualité }\end{array}$ & $\begin{array}{l}\text { Stratégie de } \\
\text { l'entreprise }\end{array}$ \\
\hline Avant 1900 & $\begin{array}{l}\text { Marché de pénurie } \\
\text { Très forte demande }\end{array}$ & $\begin{array}{l}\text { Contrôle } \\
\text { unitaire }\end{array}$ & \multirow[b]{2}{*}{$\begin{array}{l}\text { Apparition } \\
\text { implicite }\end{array}$} \\
\hline 1900-1950 & $\begin{array}{l}\text { Ere de l'industrialisation } \\
\text { Développement de la standardisation } \\
\text { Progression de la demande des ménages, }\end{array}$ & $\begin{array}{c}\text { Contrôle } \\
\text { statistique de } \\
\text { la qualité }\end{array}$ & \\
\hline 1950- 1970 & $\begin{array}{l}\text { Forte croissance, } \\
\text { Diversification des entreprises, } \\
\text { Avènement de la société de consommation }\end{array}$ & $\begin{array}{l}\text { Assurance } \\
\text { qualité }\end{array}$ & $\begin{array}{l}\text { Planification } \\
\text { stratégique }\end{array}$ \\
\hline 1970-1990 & $\begin{array}{l}\text { Période de récession, } \\
\text { Crise pétrolière, } \\
\text { Recentrage des activités des entreprises }\end{array}$ & $\begin{array}{l}\text { Total } \\
\text { Quality } \\
\text { Control }\end{array}$ & $\begin{array}{l}\text { Positionnement } \\
\text { stratégique }\end{array}$ \\
\hline 1990-2000 & $\begin{array}{l}\text { Mondialisation, } \\
\text { Concurrence exacerbée } \\
\text { Marché en saturation }\end{array}$ & \multirow{2}{*}{$\begin{array}{c}\text { Total } \\
\text { Quality } \\
\text { Management } \\
\text { Total } \\
\text { Quality } \\
\text { Learning } \\
\end{array}$} & $\begin{array}{l}\text { Management } \\
\text { Stratégique }\end{array}$ \\
\hline Récemment & $\begin{array}{l}\text { Clients bien avertis } \\
\text { Ère des nouvelles technologies et des } \\
\text { innovations }\end{array}$ & & $\begin{array}{l}\text { Modèle des } \\
\text { ressources et } \\
\text { compétences }\end{array}$ \\
\hline
\end{tabular}

Les modèles dominants qui caractérisent la démarche qualité et la stratégie des entreprises évoluent en réponses aux transformations successives de l'environnement dans lequel elles opèrent. A chaque période, un tournant se produit dans chaque discipline de manière qu'elle puisse intégrer de nouvelles approches par adaptation et/ou donner naissance à un nouveau paradigme et marquerait ainsi une nouvelle rupture. Cet examen rétrospectif de l'histoire des deux disciplines nous conforte dans l'hypothèse d'un certain déterminisme de l'évolution de la stratégie et la qualité et aussi d'une contingence à l'environnement économique et social. De plus, on peut penser 
que le fait que la stratégie et la qualité naissent presque à la même période n'est pas fortuit. C'est une période durant laquelle les deux disciplines cherchent à réaffirmer leur légitimité dans l'entreprise mais aussi dans le domaine académique. C'est ainsi qu'apparaissent les premiers signes d'une éventuelle proximité entre les deux disciplines.

Outre ce rapprochement chronologique, les proximités conceptuelles entre qualité et stratégie sont multiples. Tout d'abord, la qualité et la stratégie sont à la confluence de plusieurs disciplines. Bien qu'elles possèdent un champ de recherche clairement identifié, ces disciplines jeunes ont subi l'intérêt et se sont inspirées des autres sciences humaines et sociales. Aussi, la stratégie et la qualité se trouve au croisement de la pratique managériale et de la recherche académique. Enfin, la stratégie comme la qualité partagent des intérêts communs et des objectifs convergents.

Par ailleurs, depuis leurs premiers balbutiements jusqu'à nos jours, la démarche qualité et la stratégie tendent à élargir leur spectre de recherche pour ne pas s'enfermer dans une catégorie de problématiques bien particulières. À l'origine, le langage et la logique économique et industrielle ont été adoptés de façon majoritaire par les chercheurs en qualité et en stratégie. Cette recherche de diversité et d'adaptation à un environnement de plus en plus complexe s'accompagne d'un mouvement d'influences respectives entre la stratégie, la démarche qualité et les autres champs du domaine des sciences humaines et sociales.

Qualité et stratégie, peut-être plus que toute autre discipline, sont à l'interface entre la pratique et la théorie. Elles combinent l'optique d'action et de terrain avec celle académique. Elles connaissent un succès singulier auprès des managers, des étudiants et même auprès des néophytes en gestion. Leurs problématiques suscitent un intérêt croissant, en témoignant, le nombre de conférences et de publications qui sont toujours en progression. Les postulats des grands apports en qualité ou en stratégie sont édités dans des livres de vulgarisation, destinés à un public très large.

Le dernier facteur de rapprochement conceptuel entre qualité et stratégie tient au partage d'intérêts et d'objectifs communs. C'est aussi un sujet qui les met en rivalité. Sur ce point de vue les proximités sont nombreuses, mais nous évoquons essentiellement ceux relatifs à l'adaptation à l'environnement et à la recherche de performance.

\subsection{Démarche qualité et stratégie: Une relation consensuelle}

L'intérêt que suscite la problématique du lien démarche qualité stratégie est à l'origine de nombreux travaux. D'autant plus que le sujet est assez capricieux dans la mesure où ses implications managériales ne sont pas aussi évidentes à premier abord. On peut cependant regretter le fait que ces travaux soient en majorité anglo-saxons. Ce n'est que très récemment qu'on 
prend conscience du rôle stratégique joué par la qualité. On chemine peu à peu vers une conceptualisation du lien qualité - stratégie dans ces travaux, mais beaucoup de points obscurs restent à évoquer. On remarque aussi quelques incohérences quant à la définition des niveaux hiérarchiques où une démarche stratégique peut être envisagée, et par extension où la qualité peut avoir un apport significatif. La clarification de ces points constitue une étape nécessaire à la conceptualisation du lien démarche qualité - stratégie et à l'émergence d'une définition consensuelle de cette interface.

Ainsi, la pléthore de modèles stratégiques, pour certains relativement complexes, a créé une confusion en ce qui concerne la mise en œuvre de la qualité qui bénéficie de l'apport des normes. D'autant plus que la qualité grâce à ces dimensions éminemment stratégiques, possédé certains outils qui peuvent s'avérer pertinents pour développer une mise en œuvre simple, mais non simpliste de la stratégie.

De fait, la présence d'un système de management de la qualité au sein d'une entreprise représente une opportunité de développer une approche plus efficace de sa stratégie. Celle-ci peut alors s'appuyer sur un véritable système de management stratégique. Ce constat acquiert d'autant plus d'importance lorsque la firme a bien saisi les dimensions stratégiques de la qualité.

En outre, la qualité peut devenir un instrument de développement de l'avantage concurrentiel, lorsqu'elle crée une certaine satisfaction pour le client et/ou lorsqu'elle constitue un critère clé d'achat. La qualité est alors un moyen de différenciation qui s'apprécie selon les attentes des consommateurs et les propositions déjà existantes sur le marché (Müller W. 1991).

Dans un autre registre, la pensée stratégique s'oriente, aujourd'hui, vers la prise en compte de notions comme l'apprentissage organisationnel, et préconise davantage une approche par les ressources. Autrement dit, la stratégie est admise comme une règle pour prendre des décisions relatives au couplage de l'entreprise et de son environnement. C'est, d'ailleurs, à ce niveau, que la réflexion qualitative et celle, purement stratégique, se rejoignent. Ceci suppose que les dirigeants soient suffisamment ouverts pour admettre la stratégie et la qualité comme des vecteurs d'intégration culturelle de son entreprise. A cet effet, l'un des apports de la démarche qualité à la stratégie est de passer du diagnostic aux prescriptions. Cette étape, particulièrement délicate, définit le choix des mouvements stratégiques. Ces derniers touchent aux activités (Domaines d'Activités Stratégiques), composant le portefeuille d'activités de l'entreprise, voire à des engagements nouveaux, en termes de produits(s), marché(s) et technologie (s).

Dans ce sens, la réflexion stratégique se trouve enrichie, au moins d'un point de vue opérationnel, par les composantes de la démarche qualité. En répondant aux exigences des normes qualités, celles- ci pourvoient à l'existence et à la maintenance d'éléments universellement reconnus comme 
stratégiques. Deux d'entre eux, la segmentation et la boucle stratégique s'avèrent d'une importance particulière.

Par ailleurs, l'efficacité d'un SMQ passe tout d'abord par une politique qualité qui doit être non seulement compatible avec la stratégie de l'entreprise, mais aussi insufflée et animée par la direction17. D’un point de vue pratique, cette cohérence doit être traduite par la prise en compte des orientations et du système d'objectifs ayant guidé l'élaboration de la stratégie. La formulation de la politique qualité représente donc un acte éminemment stratégique.

\subsection{Assimilation progressive de l'orientation stratégique de la démarche qualité avec la nouvelle version de la norme ISO 9001 version 2015}

\section{1-4-1- Les nouvelles exigences de la norme ISO 9001 version 2015}

L'environnement, dans lequel les entreprises opèrent, est devenu plus complexe et plus compétitif. A cet effet, les experts du TC 176 ont décidé d'intégrer cette réalité dans la nouvelle version de la norme. Les différences les plus marquantes sont :

- La gestion de la qualité comme instrument stratégique : La norme ISO 9001 : 2015 exige aux organisations de bien décrire le contexte dans lequel elles opèrent et de se positionner par rapport à leurs concurrents. Cette contextualisation constitue ainsi le point de départ d'une approche et d'une analyse des risques ayant amené la démarche qualité à un niveau stratégique.

- La notion de «partie intéressée» constitue un fondement important de l'analyse des risques. Dans les versions précédentes de l'ISO 9001, le système de qualité était clairement axé sur le client, alors que dans la nouvelle version, tous les acteurs qui contribuent à fournir de la qualité au client sont des «partie intéressée», également les fournisseurs, le législateur, les soustraitants, etc.

- L'accent sur l'approche du processus : L'approche du processus est essentielle depuis l'apparition de la version 2000 de la norme ISO 9001. La nouvelle version de l'ISO 9001 opère ainsi une fusion de l'approche du processus et du système, jadis deux sujets distincts. En 2015, l'ISO 9004 compte que sept principes de gestion de la qualité au lieu des huit dans la version précédente.

- La forme de la nouvelle structure dite «High Level»: des huit chapitres de l'ISO 9001: 2008 sont ré-agencés en dix chapitres.

- L'amélioration au lieu d'amélioration continue : Les actions de prévention sont supprimées, et ce, en partant du principe que le système de gestion est la meilleure méthode pour prévenir les risques.

- L'absence de l'obligation «représentant de la direction»: Le texte actuel ne fait plus mention du «représentant de la direction», c'est-à-dire le

\footnotetext{
${ }^{17}$ Norme ISO 9001, chap. 5.1, Responsabilité et engagement de la direction.
} 
coordinateur qualité puisque la qualité est l'affaire de tout un chacun et de tous les niveaux au sein de l'organisation.

\section{1-4-2- L'apport de la norme ISO 9001 version 2015 dans le rapprochement} de la stratégie et la qualité

La prise de conscience des limites de la démarche qualité, progressivement renfermée dans des problématiques classiques, marque le début d'une phase de renouveau de la discipline. La démarche possède désormais un ensemble de forces et de compétences distinctives qui sont susceptibles de contribuer au renouveau et au développement d'une orientation stratégique en matière de la qualité.

L'approche du contexte, des risques et des opportunités dans la nouvelle norme ISO 9001 témoigne d'un glissement de la méthodique. Si on analyse et on gère les risques de l'entreprise par le biais de la gestion de la qualité afin de pérenniser la continuité de ses activités, on hisse alors la démarche à un niveau stratégique. Cette volonté clairement stipulée dans la nouvelle norme, ambitionne de placer la qualité autant qu'élément central du contrôle interne et de l'appréhension des risques (CHEMANGUI, 2009). La démarche renvoie dans un premier temps à l'identification, à l'évaluation, à la cotation et à la classification des risques. Dans un second temps, des actions correctives sont engagées pour prévenir les risques identifiés. Aussi, l'amélioration de la prise en compte des opportunités peut, par exemple, s'appuyer sur les modèles de diagnostic stratégique tel que l'analyse SWOT.

En outre, la notion étendue de «partie intéressée» introduite dans la nouvelle version de la norme ISO 9001 est étroitement liée et constitue un fondement important dans la réflexion stratégique. Dans la version 2008, le système qualité est clairement axé sur le client, alors que dans la nouvelle norme, tous les acteurs qui contribuent à fournir de la qualité au client sont des «partie intéressée», également les fournisseurs, le législateur, les sous-traitants, les actionnaires, etc.

\section{Des pistes théoriques pour l'analyse de l'interface stratégie-qualité : l'amélioration de la performance comme variable médiatrice}

La principale aspiration de toute entreprise est d'allouer de manière optimale les ressources nécessaires à la réalisation de ses produits afin de dégager un profit. Pour y parvenir elle utilise plusieurs mode de management, entre autre le management de la qualité qui n'est pas sans conséquences sur l'organisation. Dans ce sens, la majorité des recherches en qualité s'intéressent particulièrement à la problématique de la performance. Preuve en est le concept de l'amélioration continue (ou de l'amélioration tout court comme stipulé dans la nouvelle version de la norme ISO 9001 version 2015) sur lequel repose toute démarche qualité. Toutefois, et malgré le fait qu'on mesure depuis 
des décennies les performances en qualité à travers de nombreux indices (coût de non qualité, taux de satisfaction client, etc.), il reste encore des domaines d'investigation de la performance d'un intérêt crucial puisque la raison d'exister de la qualité réside dans sa perspective d'améliorer la performance globale de l'entreprise et c'est justement ce constat qui la rapproche du management stratégique, car l'essence même de toute stratégie est la recherche de performance. Et même les questions de facteurs clés de succès, d'avantage compétitif, de performance des décisions, de contribution à la performance globale intéressent bien aussi la qualité que la stratégie. La connaissance de l'environnement est le second point de juxtaposition des préoccupations de la démarche qualité et de la stratégie notamment dans la dernière mise à jour de la norme 9001 version 2015. Leur position dans l'entreprise et leurs connaissances sur les concurrents, les marchés et les consommateurs sont source d'amalgame des rôles, mais aussi de proximité conceptuelle évidente.

\section{La stratégie qualité : un levier de performance}

Les recherches ayant insisté sur la contribution positive de la qualité sur la performance des organisations sont nombreuses; Chan et Quazi (2002) par exemple, ont souligné que le développement d'une démarche qualité notamment de type TQM conduit toujours à une amélioration de performance. D'après les auteurs, cette dernière touche différentes dimensions à savoir : les clients, les fournisseurs, les produits et les services ainsi que les processus. L'apport des travaux de Chan et Quazi consistait à identifier un ensemble d'indicateurs de la performance ayant subi des améliorations notamment : la compétitivité, l'amélioration continue de produits et service, la réduction des coûts, l'amélioration de la productivité, la satisfaction des clients...

Un peu plutôt, l'enquête menée par Terziovski et Samson (1999) auprès de 1300 entreprises a permis d'aboutir aux conclusions suivantes :

- Les démarches qualité ont un effet significatif et positif sur la performance de l'entreprise.

- Existence de différences entre les secteurs d'activités et la taille de l'entreprise en ce qui concerne cette relation.

De sa part, Rahman (2001) a montré que la certification qualité améliore la satisfaction des clients. Elle permet ainsi de créer un avantage compétitif et par conséquent, de hausser la profitabilité.

Partant des motivations des entreprises pour la mise en place d'une démarche qualité notamment: l'augmentation de la part du marché ; la satisfaction des exigences des clients; l'amélioration de l'efficacité des processus, Withers \& Ebrahimpour (2000) ont mené une investigation sur 362 entreprises certifiées. Ils concluent que :

- La certification qualité est une source permettant à l'entreprise d'avoir un avantage compétitif. 
- Les pratiques de la qualité sont positivement corrélées avec les mesures de la performance et surtout la performance financière.

- La certification a un impact très fort sur la performance financière et opérationnelle de l'entreprise.

- Une différence remarquable en terme de performance entre les entreprises certifiées et celles non certifiées.

\section{Méthodologie}

Compte tenu de la nature spécifique de notre dispositif de recherche développé précédemment, il en ressort deux constats majeurs : Tout d'abord, le phénomène présente encore certaines ambigüités, d'où l'intérêt d'une étude exploratoire. Il doit être ensuite vérifié et modélisé (dans la mesure où nous avons souhaité mettre en évidence la corrélation entre deux variables), pour enfin être décrit et argumenté. Par ailleurs, chaque étape de l'étude empirique doit nous permettre de valider ou d'infirmer notre question de recherche.

Pour atteindre notre objectif, nous avons réalisé trois études distinctes : une étude qualitative exploratoire et deux études quantitatives avec deux collectes de données. Si certains choix méthodologiques sont communs aux deux, d'autres ne seront utilisés que pour l'une des trois études. Notre réflexion méthodologique requiert donc un examen approfondi de chaque procédure d'analyse de données.

Tableau 2 : Récapitulatif de la démarche générale de l'étude empirique

\begin{tabular}{|c|c|c|c|c|}
\hline Problématique & \multicolumn{4}{|c|}{$\begin{array}{l}\text { Quel est l'impact réciproque de la mise en place d'une démarche qualité } \\
\text { sur le processus stratégique des entreprises marocaines certifiées ISO } \\
9001 \text { ? }\end{array}$} \\
\hline Type de données & Qualitative & \multicolumn{3}{|c|}{ Quantitative } \\
\hline $\begin{array}{l}\text { Mode de recueil } \\
\text { de Données }\end{array}$ & Guide d'entretien & \multicolumn{3}{|c|}{ Questionnaire en ligne } \\
\hline $\begin{array}{l}\text { Méthode de } \\
\text { collecte }\end{array}$ & $\begin{array}{l}\text { Conversation } \\
\text { téléphonique }\end{array}$ & \multicolumn{3}{|c|}{ Diffusion en ligne } \\
\hline Période d'étude & \multicolumn{4}{|c|}{$2015-2017$} \\
\hline Population & $\begin{array}{l}\text { Experts et stratégie- } \\
\text { qualité }\end{array}$ & \multicolumn{3}{|c|}{$\begin{array}{l}\text { Entreprises marocaines certifiées ISO } 9001 \text { (969 } \\
\text { entreprises en 2015) }\end{array}$} \\
\hline $\begin{array}{c}\text { Objet de la } \\
\text { phase }\end{array}$ & Etude exploratoire & $\begin{array}{l}\text { Analyse } \\
\text { exploratoire }\end{array}$ & $\begin{array}{l}\text { Analyse } \\
\text { confirmatoire }\end{array}$ & $\begin{array}{l}\text { Test des } \\
\text { modèles }\end{array}$ \\
\hline Logiciels utilisés & NVIVO & SPSS & $\begin{array}{ll}\text { SPSS } & + \\
\text { AMOS } & \end{array}$ & AMOS \\
\hline $\begin{array}{c}\text { Taille de } \\
\text { l'échantillon }\end{array}$ & 19 Experts & 46 entreprises & 97 entr & eprises \\
\hline $\begin{array}{c}\text { Technique } \\
\text { d'échantillonnag } \\
\text { e }\end{array}$ & \multicolumn{4}{|c|}{ par convenance } \\
\hline $\begin{array}{c}\text { Techniques } \\
\text { d'analyse de } \\
\text { données }\end{array}$ & $\begin{array}{l}\text { Analyse du contenu } \\
\text { thématique }\end{array}$ & ACP & $\begin{array}{c}\text { ACP + } \\
\text { Equations } \\
\text { structurelles }\end{array}$ & $\begin{array}{l}\text { Equations } \\
\text { structurelles }\end{array}$ \\
\hline
\end{tabular}




\subsection{Explorer les modalités du lien stratégie-qualité}

Le bien-fondé de notre question de recherche peut être évalué en fonction des particularités du terrain d'étude notamment celui des entreprises marocaines, de la pertinence des résultats obtenus, mais surtout du degré de validité et de fiabilité de la démarche poursuivi.

Dans ce sens, en raison de la complexité de décrire avec exactitude la nature de la relation entre la stratégie et la qualité chez les entreprises marocaines, nous avons jugé nécessaire de mener au préalable une étude exploratoire, dans la mesure où la revue de littérature nous a peu renseignés sur la nature de cette interface. Dans cette perspective, une enquête menée à partir d'un guide d'entretiens administré auprès de 19 experts et consultants dans les domaines de la stratégie et la qualité a été envisagée.

L'analyse de contenu thématique réalisée sur le corpus des récits oraux des répondants collectés grâce à un guide d'entretien, dont nous avons transcrit les principales idées, au moyen du logiciel NVivo a débouché sur l'expression plus précise de la problématique en limitant notre champ d'investigation aux entreprises disposant d'un système de management qualité et en introduisant l'amélioration de la performance comme variable médiatrice dans cette relation. Elle a permis aussi de générer des énoncés dans une perspective de création d'échelles de mesure relatives aux modalités d'interférence entre la stratégie et la qualité qui n'existeraient pas dans la littérature. Ainsi, en plus des variables issues de l'analyse de la littérature, nous avons identifié treize échelles de mesure dont cinq relatives au processus stratégique, deux portant sur le comportement qualité des entreprises et sept échelles de mesure décrivant l'interface stratégie-qualité. Ces différentes échelles de mesure nous en servi pour enrichir notre modèle initial.

\section{Figure 1 : Modèle de recherche enrichi}

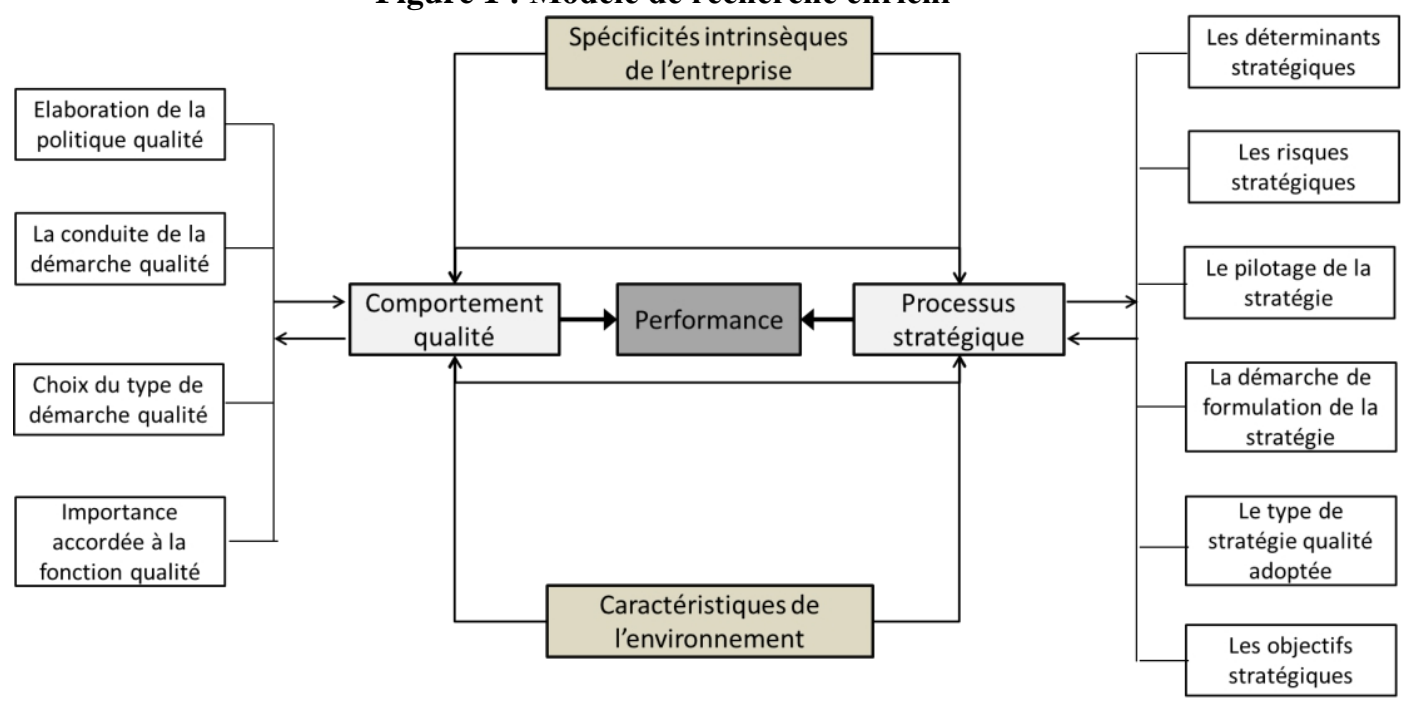




\subsection{Valider les énoncés des instruments de mesure du lien stratégie/ qualité}

Si l'analyse exploratoire a pour objet de contribuer à générer des énoncés dans une perspective de création d'échelles de mesure qui n'existeraient pas dans la littérature, la deuxième étape empirique consiste quant à elle, à l'organisation de ces instruments de mesure en questionnaire et puis la mobilisation d'une enquête auprès des entreprises marocaines afin de vérifier la fiabilité et la validité de ces échelles de mesure et in fine, de tester le modèle de recherche enrichi.

L'utilisation d'une enquête par questionnaire résulte d'une réflexion sur la manière d'effectuer la phase quantitative de notre recherche. Lors de la création des échelles de mesure, notre objectif était donc de réduire au maximum les erreurs systématiques (biais provenant de la conception de l'instrument) et aléatoires (biais provenant des circonstances liées à l'étude). Pour ce faire, Churchill (1979) propose une méthode qualifiée de paradigme méthodologique pour développer des échelles d'attitudes. Dans cette perspective, la conduite d'une étude de la dimensionnalité des construits à travers une analyse factorielle sur un échantillon de 46 entreprises marocaines certifiées ISO 9001 nous a permis de mettre en évidence les dimensions essentielles à la compréhension du phénomène et de purifier l'instrument de mesure tel que défini dans le paradigme de Churchill.

Afin de déterminer la structure factorielle des échelles, nous avons eu recours à des analyses en composantes principales (ACP). Les résultats de cet ACP a fait l'objet d'une procédure de validation en utilisant les méthodes d'équations structurelles. Cette approche requiert selon Churchill une nouvelle collecte de données auprès cette fois-ci de 97 entreprises marocaines certifiées ISO 9001. Si l'analyse exploratoire a pour objet de contribuer à générer des énoncés dans une perspective de création d'échelles de mesure qui n'existeraient pas dans la littérature, la deuxième étape empirique consiste quant à elle, à l'organisation de ces instruments de mesure en questionnaire et puis la mobilisation d'une enquête auprès des entreprises marocaines afin de vérifier la fiabilité et la validité de ces échelles de mesure et in fine, de tester le modèle de recherche enrichi.

L'utilisation d'une enquête par questionnaire résulte d'une réflexion sur la manière d'effectuer la phase quantitative de notre recherche. Lors de la création des échelles de mesure, notre objectif était donc de réduire au maximum les erreurs systématiques (biais provenant de la conception de l'instrument) et aléatoires (biais provenant des circonstances liées à l'étude). Pour ce faire, Churchill propose une méthode qualifiée de paradigme méthodologique pour développer des échelles d'attitudes. Dans cette perspective, la conduite d'une étude de la dimensionnalité des construits à travers une analyse factorielle sur un échantillon de 46 entreprises marocaines certifiées ISO 9001 nous a permis de mettre en évidence les dimensions 
essentielles à la compréhension du phénomène et de purifier l'instrument de mesure tel que défini dans le paradigme de Churchill.

Afin de déterminer la structure factorielle des échelles, nous avons eu recours à des analyses en composantes principales (ACP). Les résultats de cet ACP a fait l'objet d'une procédure de validation en utilisant les méthodes d'équations structurelles. Cette approche requiert selon Churchill une nouvelle collecte de données auprès cette fois-ci de 97 entreprises marocaines certifiées ISO 9001.

\subsection{Identifier la nature et les déterminants de la relation stratégie-qualité}

Cette étape empirique ultime, constitue le socle de notre recherche. Elle consiste en le test des modèles préalablement établis. Ainsi, nous vérifions en premier lieu les modèles relatifs à l'importance des spécificités intrinsèques de l'entreprise et des caractéristiques de son environnement dans la définition de ces choix stratégiques et de son comportement qualité. Dans un second lieu, nous testons les modèles de la relation de causalité directe entre la stratégie et la qualité. Pour enfin, valider le rôle médiateur de la performance dans l'explication de la relation stratégie-qualité. Pour ce faire, nous utilisons encore une fois, et sous un autre angle, la méthode des équations structurelles pour le test de ces différents modèles.

\section{Principaux résultats de l'étude empirique}

\subsection{Constitution de l'échantillon pour le test du modèle enrichi}

La constitution d'un échantillon représentatif n'est pas une fin en soi. Ce que nous désirons c'est que les résultats issus du traitement statistique de l'échantillon puissent être « extrapolés » à la population. Par conséquent, ce que l'on cherche c'est plutôt que les résultats soient « représentatifs » (Dépelteau, F. 2000).

Dans notre cas, nous avons recensé auprès de l'Organisation Internationale de Normalisation 969 entreprises marocaines certifiées ISO 9001 qui représentent notre population d'étude. Toutefois, l'absence d'une liste exhaustive unique des entreprises marocaines certifiées ISO 9001 a rendu difficile l'identification précises de ces entreprises. C'est pourquoi nous avons procédé à des recoupements entre plusieurs sources d'informations :

- L'annuaire de la délégation provinciale de l'industrie, de l'investissement, du commerce et de l'économie numérique ;

- Le portefeuille client de certains cabinets de certification (SGS-Maroc et bureau Véritas) ;

- Les listes publiées sur internet relatives aux entreprises marocaines certifiées.

De ces différentes bases de données, nous avons procédé à un échantillonnage aléatoire pour la sélection des entreprises enquêtées. Au final, 
nous nous sommes parvenu à l'administration de 97 questionnaires auprès des entreprises marocaines certifiées ISO 9001.

5.2. Modèle global de l'interface stratégie-qualité chez les entreprises marocaines

Nous avons entamé le processus de validation de notre modèle général par la vérification de l'impact réciproque de la réflexion stratégique sur la pratique qualité chez les entreprises avant d'examiner le rôle des caractéristiques de l'entreprise et de l'environnement dans la définition de cette relation.

Dans un premier temps, la lecture de la sortie des résultats issus des modèles dérivés est centrée sur la vérification de l'absence de variances négatives et de coefficients standardisés supérieurs à 1 . L'édition des résultats par le logiciel AMOS indique que ces conditions minimales sont remplies.

Après vérification de la significativité des relations entre les différentes dimensions de notre modèle, l'examen de la qualité d'ajustement du modèle affirme la bonne qualité des différents modèles dérivés18. En revanche, les autres indices (GFI, AGFI, CFI) n'atteignent pas les seuils acceptables. Dès lors, nous considérons que le modèle est acceptable dans sa globalité et qu'il s'ajuste suffisamment bien aux données observées compte tenu de sa complexité. abouti :

En définitif, la figure suivante reprend le modèle auquel nous avons Figure 2 : Modèle global de l'interface stratégie-qualité chez les entreprises marocaines

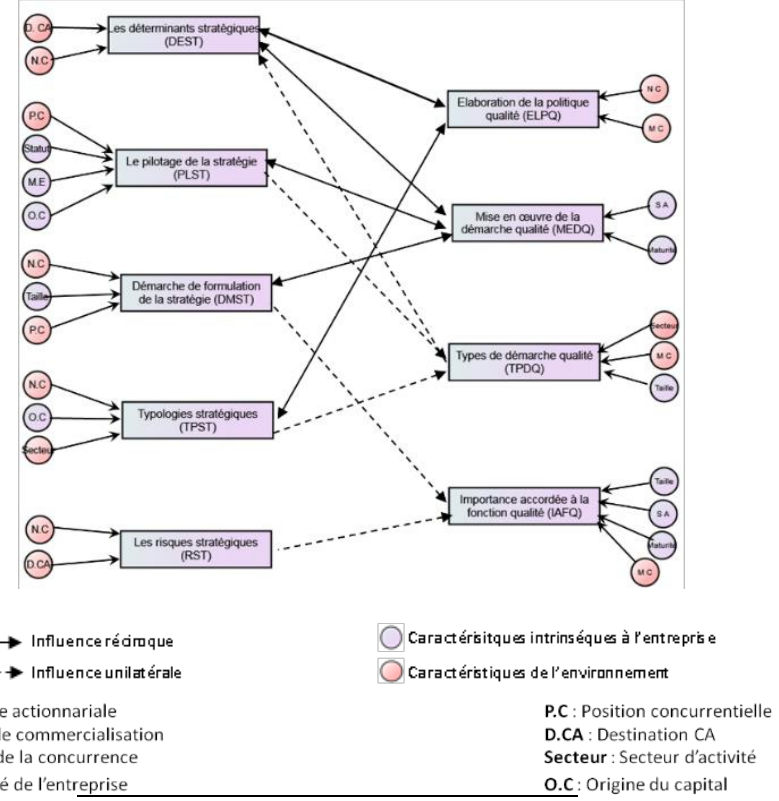

${ }^{18} \chi^{2} / \mathrm{ddl}$ est inférieur au seuil de 5 ; le RMSEA est faible $(=0,08)$ et le CAIC est inférieur à celui du modèle saturé. 
Les résultats des équations structurelles appliquées aux modèles de l'interface stratégie-qualité sont dans leurs ensembles satisfaisants.

Ainsi, le processus stratégiques des entreprises marocaines est déterminé par « la maturité de l'entreprise », « sa taille », « son statut » et « l'origine de son capital». Il est aussi conditionné par certaines caractéristiques de l'environnement à savoir: «le secteur d'activité », «la nature de la concurrence », « la position concurrentielle » et «la destination du chiffre d'affaires $\gg$.

De sa part, le comportement qualité est dépendant de « la maturité de l'entreprise », de « sa taille » et de sa « structure actionnariale ». Il est aussi influencé par les caractéristiques du « secteur d'activité », la «nature de la concurrence » et « le mode de commercialisation ».

Par ailleurs, pour ce qui de l'influence réciproque de la stratégie et de la démarche qualité, les analyses des régressions effectuées au moyen des modèles d'équations structurelles révèlent que toutes les dimensions de la stratégie influencent de manière significative au moins un des volets du comportement qualité des entreprises. En revanche, pour l'autre sens de la relation (Démarche qualité $\rightarrow$ Stratégie), les tests des équations structurelles ont affirmé l'influence significative de trois volets du comportement qualité de l'entreprise sur la conduite de sa stratégie (La politique qualité, la mise en œuvre du projet qualité et le type de démarche qualité implantée). Ces résultats relativement bruts méritent que l'on s'y attarde quelques instants.

Tout d'abord, nous constatons que la qualité globale des régressions est assez élevée. Toutefois, si la stratégie de l'entreprise explique $64 \%$ de la variance de son comportement qualité, alors que réciproquement, ce dernier n'explique que $47 \%$ de la stratégie. Ces résultats sont tout à fait satisfaisants. Ils indiquent que les relations mises en évidence entre la stratégie et la démarche qualité, malgré la différence de poids, sont réellement porteuses de sens. Aussi, nous avons démontré que les déterminants d'élaboration de la stratégie portant principalement sur « les clients, le contexte environnemental et sociétal, les concurrents et les actionnaires » semblent inspirer le contenu de la politique qualité ainsi que l'ampleur des actions mise en œuvre dans le cadre du projet qualité de l'entreprise (gestion des risques, études de satisfaction clients, étude de satisfaction du personnel). Nous avons constaté aussi que les risques stratégiques, manifestés dans la rareté de la MOD, les changements des tendances et goûts des clients, la difficulté d'approvisionnement et la concurrence, conditionnent fortement la place et le poids de la fonction qualité dans l'entreprise. En effet, plus l'entreprise se trouve confronter à un environnement turbulent plus elle a tendance a donné de l'importance et du poids à la fonction qualité au sein de la structure (effectif, nature de la structure, position dans l'organigramme...). Autrement dit, il s'agit pour ces entreprises d'un outil de gestion des risques. Egalement, le pilotage de la stratégie (par 
l'entreprise mère, ou en interne) détermine significativement aussi bien le type de démarche qualité adoptée (SMQ, SME, OHSAS, TQM...) que les actions qualité mise œuvre. Quant à la démarche de formulation de la stratégie, elle influence la mise en œuvre de la démarche qualité ainsi que la place de la fonction chargée de la qualité au sein de l'entreprise. Enfin, les tests ont indiqué que le type de stratégie adoptée oriente le choix du type de la démarche qualité mise en œuvre ainsi que le contenu de la politique qualité.

\subsection{Etude du rôle médiateur de la performance dans la définition de l'interface stratégie-qualité}

Aussi bien dans la littérature, que dans les travaux empiriques, nous avons soulevé beaucoup de débats sur le l'impact éventuel des actions stratégiques notamment celles relatives à la mise en place d'une démarche qualité sur l'amélioration de la performance des entreprises. Notre apport à ce niveau consiste alors a confirmé ou infirmé cette relation dans le contexte des entreprises marocaines certifiées. Par ailleurs, et compte tenu la polysémie de la notion de performance, nous avons développé lors de cette recherche une échelle de mesure propre à l'échantillon étudié.

L'édition des résultats par le logiciel AMOS indique l'absence de variance négative et de coefficients standardisés supérieurs à 1 . Les conditions minimales d'acceptation du modèle sont alors remplies.

Les tests $\mathrm{t}$ de Student sont en général significatifs à l'exception de certaines échelles qui ont été retirées pour améliorer la qualité de notre modèle.

Pour ce qui est de la qualité d'ajustement, le $\chi^{2} / \mathrm{ddl}$ et le CAIC présentent des valeurs satisfaisantes. Les autres indices (GFI, AGFI, RMSEA, CFI) quant à eux n'atteignent pas les seuils acceptables. Toutefois, ils sont suffisants au regard des critères aménagés pour les modèles complexes.

A partir des tests réalisés, il s'avère que la performance est bien une variable médiatrice dans la relation entre la stratégie et la démarche qualité dans l'entreprise. Toutefois, cet effet médiateur n'est que partiel, dans la mesure où les coefficients de régression entre certains échelles de mesure de la stratégie et la qualité d'une part et la performance d'autre part restent non significatifs.

Figure 3 : Modèle relatif au rôle médiateur de la performance dans le rapprochement de la stratégie et la démarche qualité dans l'entreprise

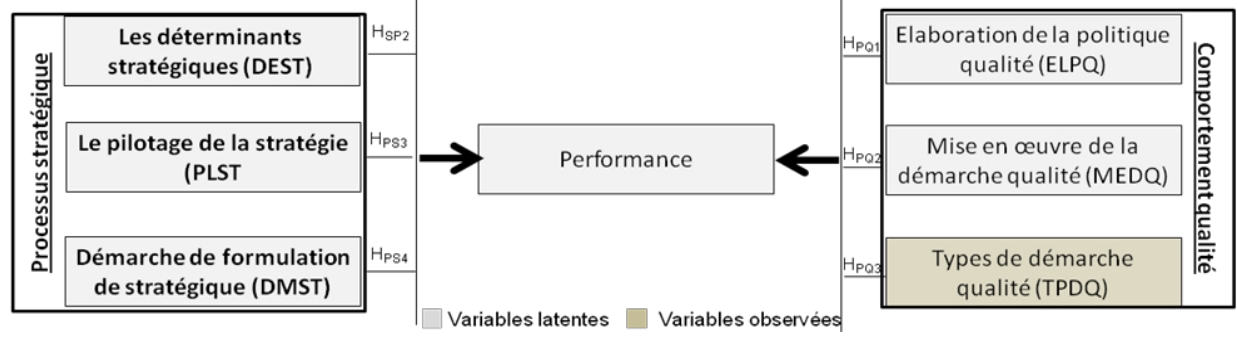


La stratégie et la qualité permettent alors à l'entreprise d'assurer une certaine maitrise et de tirer profit de son environnement en fondant un avantage concurrentiel lui permettant de générer de la valeur compte tenue de ces capacités.

L'importance accrue pour l'obtention d'un avantage concurrentiel peut constituer le point de départ du processus de rapprochement entre la stratégie et la démarche qualité. Dans cette optique, selon Caby, Hirigoyen (2001), «trois types de leviers d'essence stratégique sont à l'origine de l'avantage concurrentiel source de création de valeur : les leviers stratégiques stricto sensu, des leviers financiers et des leviers de gouvernance d'entreprise ». Dès lors, la démarche qualité comme choix stratégique ou même comme levier de gouvernance peut constituer un avantage concurrentiel pour l'entreprise.

Figure 4 : Rôle de la démarche qualité dans l'obtention d'un avantage concurrentiel

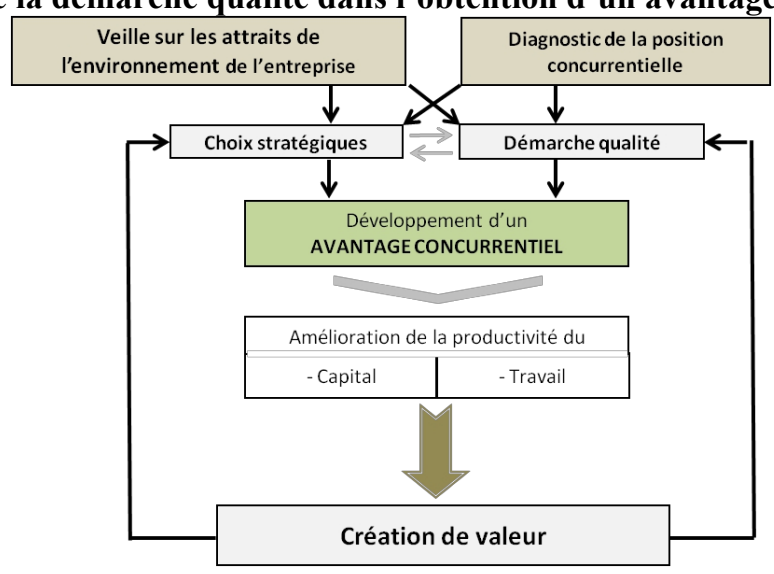

Deux conceptions majeures sont alors à l'origine de la contribution de la démarche qualité à l'avantage concurrentiel de l'entreprise. Une approche classique issue des travaux de Porter qualifiée de "distributive» selon laquelle, l'entreprise certifiée développe des facteurs clés de succès en rassurance voire modelant son environnement et qui vont lui permettre d'exploiter la confiance des clients à l'encontre d'autres acteurs.

Ces facteurs clés de succès concernent les atouts détenus par l'entreprise en termes de ressources et de compétences qui lui procurent un avantage concurrentiel non imitable à court terme. Cinq grandes catégories de critères sont généralement distinguées à ce niveau : la position sur le marché, la structure des coûts, l'image, les compétences techniques et l'innovation.

La deuxième conception dite " contributive 》 développée à partir des années 97, suppose que grâce à la mise en place d'une démarche qualité, l'entreprise pourrait bien créer davantage de richesses par un meilleur usage 
de ses ressources. Elle procure ainsi à l'entreprise une capacité dynamique pour son développement.

Ces deux points de vue ne sont nullement exclusifs mais complémentaires pour justifier la diversité des types de démarches qualité observés chez les entreprises de notre échantillon.

Les facteurs clés de succès concernent les atouts détenus par l'entreprise en termes de ressources et de compétences qui lui procurent un avantage concurrentiel non imitable à court terme. Cinq grandes catégories de critères sont généralement distinguées à ce niveau : la position sur le marché, la structure des coûts, l'image, les compétences techniques et l'innovation.

La deuxième conception dite « contributive » développée à partir des années 97, suppose que grâce à la mise en place d'une démarche qualité, l'entreprise pourrait bien créer davantage de richesses par un meilleur usage de ses ressources. Elle procure ainsi à l'entreprise une capacité dynamique pour son développement. Ces deux points de vue ne sont nullement exclusifs mais complémentaires pour justifier la diversité des types de démarches qualité observés chez les entreprises de notre échantillon.

\section{Des pistes d'interprétation totalement cohérentes avec le cadre de notre recherche.}

Les résultats auxquels nous avons abouti dans le cadre de notre étude empirique rejoint globalement les apports théoriques et les travaux empiriques exposés en première partie de cette thèse. Ce qui nous a permis de s'y référer pour en tirer des interprétations pertinentes.

Dans une première perspective, la relation stratégie et la démarche qualité trouve ses arguments dans d'une part, le rôle de la démarche qualité comme vecteur de transformation, outil de pilotage et de management des risques pour l'entreprise. Et d'autre part, du fait que la qualité est considérée comme une déclinaison de la stratégie.

Dans une autre perspective, nous prétendons que le succès de l'interface découle de l'obtention par l'entreprise d'un avantage concurrentiel grâce au développement d'un certain nombre de facteurs clés de succès et de capacités dynamiques contribuant à l'amélioration de la performance des entreprises.

Par ailleurs, nous avons justifié inefficience d'une stratégie qualité grâce à l'extrapolation des enseignements du modèle de "l'alignement stratégique " à l'interface stratégie qualité. En effet, seule une relation harmonieuse et cohérente entre la stratégie générale de l'entreprise et la démarche qualité mise en œuvre et disposant de ressources suffisantes pour atteindre l'objectif visé générerait effectivement plus de performance. Ainsi, et comme nous l'avons déjà évoqué plus haut, recourir à une stratégie qualité 
procure à l'entreprise de nouveaux facteurs clés de succès et renforce sa capacité dynamique, génère l'avantage concurrentiel et donc crée de la valeur.

Toutefois, ces retombées ne sont pas aussi automatiques. Ce constat, nous ramène donc à affiner notre réflexion afin de comprendre le mécanisme de fonctionnement de la stratégie et de la démarche qualité permettant à l'entreprise de créer effectivement de la valeur. Dans ce sens, nous nous référons au modèle de " l'alignement stratégique » pour répondre à notre questionnement.

\section{Conclusion}

Au terme de cette recherche, il est désormais opportun de dresser un modèle efficient de l'interface stratégie-qualité conformément aux enseignements théoriques et empiriques auxquels nous avons aboutis.

Ainsi, et tout comme le souligne M. Porter (1980), l'analyse de l'environnement dans lequel opère l'entreprise la conduit à élaborer, en tout premier lieu, sa stratégie d'où découlent les objectifs stratégiques. Par la suite, des politiques viennent préciser la façon dont les objectifs stratégiques seront opérationnalisés y incluant ceux faisant partie des démarches qualité à mettre en œuvre. Des choix seront effectués grâce par exemple à la chaîne de Porter puis définis (par exemple : cartographie des processus) de sorte qu'il sera possible, par la suite, d'intégrer des indicateurs de performance appropriés. Ces indicateurs de performance reflèteront à la fois les exigences des clients des processus et les attentes relatives aux objectifs stratégiques initiaux.

Une fois les résultats de ces indicateurs de performance connus, ils sont comparés à leur cible respective puis communiqués aux différents paliers hiérarchiques pour leurs besoins respectifs. Et c'est alors que la boucle de l'amélioration continue fait son œuvre.

Ce schéma global aidera les dirigeants des entreprises ayant opté pour une démarche qualité à définir leurs processus d'affaires, identifier leurs indicateurs de performance puis les intégrer aux processus.

Si cette démarche globale est bien mise en œuvre, toutes les conditions de performance seront réunies. En résumé, nous pouvons schématiser l'interface démarche qualité - stratégie d'entreprise selon un processus séquentiel adossé au cycle PDCA. 
Figure 5 : Modèle général de l'interface stratégie-qualité

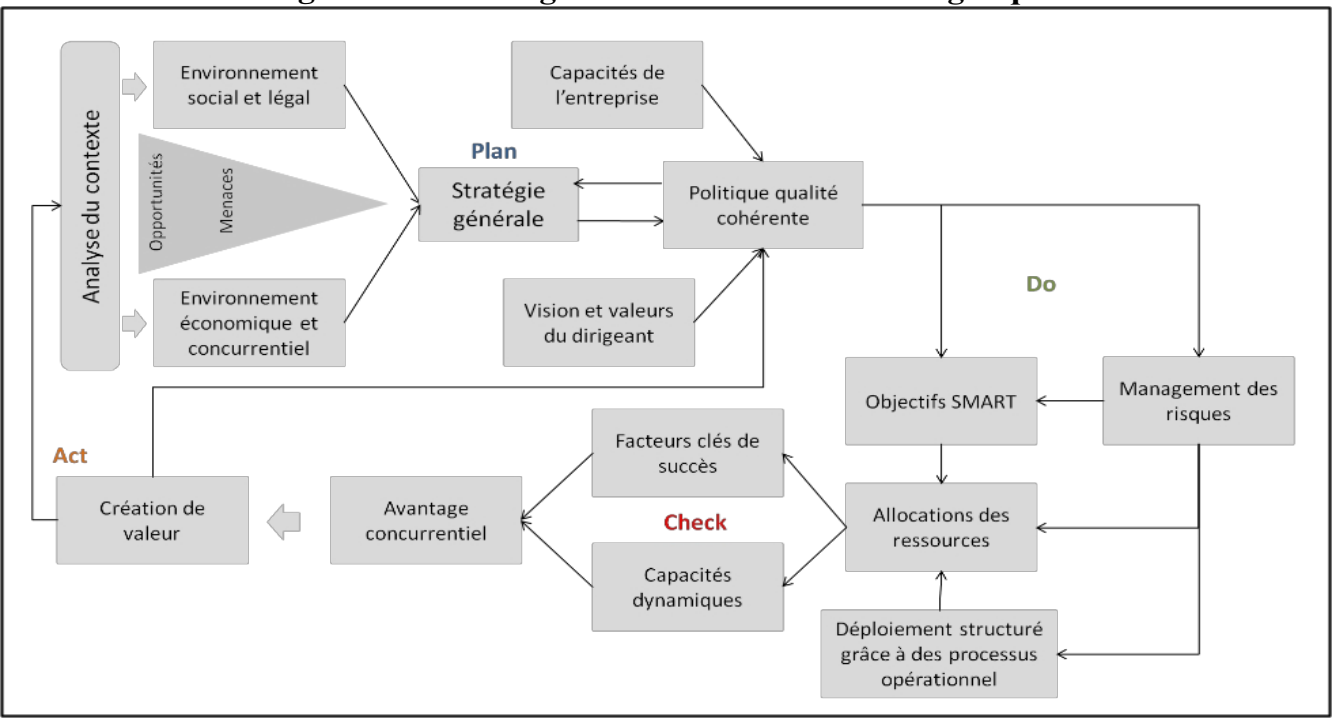

\section{References :}

1. Benezech D., \& Loos-Baroin J., (2003), Le processus de certification ISO 9000 comme outil d'apprentissage organisationnel, Revue Sciences de gestion, $n^{\circ} 36$, pp 11-41.

2. Caby J. et Hirigoyen G. (2001), La création de valeur de l'entreprise, $2^{\text {ème }}$ édition, Economica.

3. Chan, T. H., Quazi, H. A. (2002), Overview of quality management practices in selected Asian countries. Quality Management Journal, 9 (1), pp 172-180.

4. Churchill G.A, (1979), A paradigm for developing better measures of marketing constructs ", Journal of Marketing Research, vol.16, February, 64-73.

5. Chemangui, M. (2009) «Proposition d'une métrique de la qualité de l'audit : expérimentation dans le cadre des relations d'agence internes ». Comptabilité, Contrôle Audit vol 1, 15, pp225-248.

6. Dépelteau, F. (2000), La démarche d'une recherche en sciences humaine, Bruxelles, Éditions De Boeck Université.

7. Evans, J.R., (2004) An exploratory study of performance measurement systems and relationships with performance results. Journal of Operations Management, 22 (3): pp 219-232.

8. Khetib, M. \& Ghomari, S. « L'Impératif du Management de la Qualité pour les Entreprises Algériennes face aux Défis de la Mondialisation » Colloque international Management de la qualité totale \& Développement de la performance de l'entreprise, Université Dr Tahar Moullay Saida 2010. 
9. Lambert G., Loos-Baroin J., (2004), Certification ISO 9000 et création de connaissances opérationnelles ou conceptuelles: une étude de cas, Revue Finance - contrôle - stratégie, Vol. 7, n`1 pp 53-79.

10. Müller W. (1991). Gaining competitive advantage through customer satisfaction, European Management Journal, V9 $n^{\circ} 2$, Juin pp 201-210.

11. Porter M.E. (1980), Competitive Strategy, Techniques for Analysing Industries and Competitors. Free Press, New York.

12. Rahman, S. (2001), A comparative study of TQM practice and organizational performance of SMEs with and without ISO 9000 certification, International Journal of Quality and Reliability Management, Vol 18, 1 pp 35-49.

13. Svetoslav Georgiev \& Emil Georgiev (2015) "Motivational Factors for the Adoption of ISO 9001 Standards in Eastern Europe: The Case of Bulgaria", Journal of Industrial Engineering and Management, 8 (3), pp 1020-1050.

14. Siegel, D. Roesslinge, F. (2015) "Management stratégique et management de la qualité", AFNOR Editions.

15. Terziovski, M. \& Samson, D. (1999) The link between total quality management practice and organizational performance. International Journal of Quality \& Reliability Management, 16 (3) pp. 226-237.

16. Withers, B., Ebrahimpour, M. (2000) "Does ISO 9000 certification affect the dimensions of quality used for competitive advantage?" European Management Journal vol 18, $N^{\circ}$ 4, pp 431-443. 